\begin{tabular}{|c|l|}
\hline Title & Correlations and spin flips in tunneling through a quantum dot \\
\hline Author(s) & A kera, Hiroshi \\
\hline Citation & $\begin{array}{l}\text { Physical Review B, 59/15), 9802-9805 } \\
\text { https://doi.org/10.1103/PhysRevB.59.9802 }\end{array}$ \\
\hline Issue Date & 1999-04_15 \\
\hline Doc URL & http://hdl.handle.net/2115/6059 \\
\hline Rights & Copyright $\odot 1999$ A merican Physical Society \\
\hline Type & article \\
\hline File Information & PRB59_15.pdf \\
\hline
\end{tabular}

Instructions for use 


\title{
Correlations and spin flips in tunneling through a quantum dot
}

\author{
Hiroshi Akera \\ Department of Applied Physics, Hokkaido University, Sapporo 060-8628, Japan \\ and Institute for Solid State Physics, University of Tokyo, Roppongi, Tokyo 106-8666, Japan
}

(Received 26 October 1998)

\begin{abstract}
Variations of the Coulomb oscillations with the in-plane magnetic field (the spin splitting) are theoretically studied to find many-body effects in tunneling through a quantum dot. The conductance and the transmission coefficient are calculated at small tunneling rates in the absence of spin-orbit interactions and expressed in terms of universal functions of the spin splitting, which have no dependence on details of wave functions except the magnitude of spin. Such universal functions are shown to be useful in observing the correlation between tunnelings of electrons with opposite spins and the dephasing due to spin flips.
\end{abstract}

[S0163-1829(99)11815-0]

Quantum dots provide an almost unparalleled opportunity to study many-body effects in tunnelings. A typical phenomena is a set of sharp peaks of the conductance that appear periodically as a function of the gate voltage (Coulomb oscillations). ${ }^{1,2}$ The Coulomb oscillations are due to the Coulomb blockade: if one electron enters the dot at a gate voltage, the Coulomb repulsion blocks another to enter the dot until the gate voltage is raised and overcomes the Coulomb repulsion. This Coulomb blockade appears also in the conductance peak height, since electron tunnelings are correlated and the current is reduced. Such a correlation effect on the peak height has been difficult to observe because the peak height is subject to details of the potential profile in a quantum dot and tunneling barriers, which is not known at desired accuracy.

Glazman and Matveev $^{3}$ studied theoretically the magnetic-field dependence of the current through resonance centers in a barrier of tunnel junctions. They calculated the tunneling current using the kinetic equations for a resonance center with a single orbit of small radius, in which the orbit has little dependence on the magnetic field. In the absence of the magnetic field, two spin levels are degenerate, but electrons with opposite spins cannot tunnel into the dot simultaneously due to the Coulomb correlation, giving rise to a reduction in the current. When the spin splitting is large enough, on the other hand, only one spin level is used for the current, and the correlation effect disappears. Therefore the correlation is reflected in the magnetic-field dependence of the conductance and has been observed in the experiment. ${ }^{4}$

In this paper we extend the Glazman-Matveev theory to transport through a quantum dot with many orbits and show that the magnetic field is a useful tool to clarify the Coulomb correlation as well as the dephasing due to spin flips at such a quantum dot. The magnetoconductance is calculated for the magnetic field parallel to the two-dimensional plane and is shown to be universal in the sense that it has no dependence on details of tunneling matrix elements and many-body wave functions in the dot. Important assumptions used in its derivation are (i) the in-plane magnetic field affects only the spin degree of freedom in our two-dimensional electron systems with negligible spin-orbit interactions and (ii) the tunneling is weak so that the level broadening is much smaller than the thermal energy. In the conductance peak associated with the transition between the $N$-electron ground state (spin $S$ ) and the $N+1$-electron ground state $(\operatorname{spin} \widetilde{S})$, the normalized conductance is a universal function that depends only on $S, \widetilde{S}$, the spin splitting and the chemical potential divided by the thermal energy.

In the presence of interactions in a quantum dot, there are tunneling processes in which the spin of a tunneling electron is flipped and at the same time the spin of the dot is changed. Such a spin flip at the quantum dot is another fundamental many-body effect and affects the phase coherence of the current through the dot. The coherence in the electron wave through a quantum dot has been studied theoretically ${ }^{5-12}$ and experimentally ${ }^{13-15}$ employing the Aharonov-Bohm effect in a system with a dot. The recent experiment in a double-slit geometry ${ }^{15}$ has successfully obtained the coherent transmission coefficient through the dot, but no experiments have observed the dephasing due to spin-flip processes at the dot. In this paper we show that the transmission coefficient is also represented by a universal function of the spin splitting, which exhibits the existence of spin-flip scatterings in a simple manner.

Our Hamiltonian $H$ consists of $H_{d}$ for the dot, $H_{L}$ for the leads, and $H_{t}$ for tunnelings:

$$
\begin{gathered}
H=H_{d}+H_{L}+H_{t}, \\
H_{\mathrm{d}}=\sum_{n \sigma} \varepsilon_{n \sigma} c_{n \sigma}^{\dagger} c_{n \sigma}+H_{\mathrm{int}}, \\
H_{\mathrm{L}}=\sum_{l k \sigma} \varepsilon_{l k \sigma} c_{l k \sigma}^{\dagger} c_{l k \sigma}, \\
H_{\mathrm{t}}=\sum_{l k n \sigma}\left(V_{l k n} c_{l k \sigma}^{\dagger} c_{n \sigma}+\text { H.c. }\right),
\end{gathered}
$$

where $c_{n \sigma}^{\dagger}\left(c_{n \sigma}\right)$ are creation (annihilation) operators for an electron with spin $\sigma$ in orbit $n$ in the dot and $c_{l k \sigma}^{\dagger}\left(c_{l k \sigma}\right)$ are those for an electron in state $k$ in lead $l(l=e$ for the emitter and $l=c$ for the collector). $H_{\text {int }}$ is the interaction term whose explicit form is not necessary in the following general dis- 
cussion. We only need to assume that the energy separation between the ground-state multiplet and the first excited state for $N$ electrons in the dot and that for $N+1$ electrons are larger than the thermal energy, so that the current is exclusively due to transitions between the ground states for the two electron numbers. Each state in the ground-state multiplet (spin $S$ ) is labeled by the $z$ component of the spin $S_{z}$.

In the weak-tunneling regime we consider, the level broadening due to tunneling is much smaller than the thermal energy, so that electronic states and electron distribution in leads are little modified by tunnelings and correlations between the leads and the dot, such as in the Kondo effect, can be neglected. In this regime we can calculate the linear conductance by applying our previous theory, ${ }^{16}$ which employs the transition-rate formula for electron tunnelings between the two leads by incorporating the finite level broadening of intermediate many-body states. We employ this method here, first because it is simpler than the general current formula with the Keldysh-Green functions, ${ }^{17}$ and, second, because it is applicable also to the off-resonance regime discussed later (the latter is not the case in the formulation using the Master equation, or the kinetic equation ${ }^{18,19}$ ).

In calculating the transition rate, the initial state is $|i\rangle$ $=\left|e k \sigma, L ; N, S S_{z}\right\rangle$ in which $e k \sigma$ state is occupied and $|L\rangle$ represents the occupation of the other levels in the two leads.

One of the intermediate states is $|m\rangle=\left|L ; N+1, \widetilde{S} \widetilde{S}_{z}\right\rangle$ with $\widetilde{S}_{z}=S_{z}+\sigma / 2$. The final state is $|f\rangle=\left|c k^{\prime} \sigma^{\prime}, L ; N, S S_{z}^{\prime}\right\rangle$ with $S_{z}^{\prime}+\sigma^{\prime} / 2=S_{z}+\sigma / 2$. The transition rate is given by

$$
P_{i, f}=\frac{2 \pi}{\hbar}|\langle i|\hat{T}| f\rangle|^{2} \delta\left(E_{i}-E_{f}\right)
$$

with

$$
\langle i|\hat{T}| f\rangle=\left\langle i\left|H_{t}\right| m\right\rangle\langle m|G| m\rangle\left\langle m\left|H_{\mathrm{t}}\right| f\right\rangle .
$$

The propagator is defined by $G=\left(E_{i}-H+i \eta\right)^{-1}$ with $\eta$ the positive infinitesimal. $\langle m|G| m\rangle$ is replaced by its thermal average with respect to $|L\rangle$ and evaluated in the noncrossing approximation, ${ }^{20,21}$ which is accurate, at least, in the weaktunneling regime.

The current is then expressed as

$$
\begin{aligned}
I= & -e \sum_{k \sigma S_{z}} \sum_{k^{\prime} \sigma^{\prime} S_{z}^{\prime}}\left[P_{i, f} f_{e k \sigma}\left(1-f_{c k^{\prime} \sigma^{\prime}}\right) g_{N S_{z}}\right. \\
& \left.-P_{f, i} f_{c k^{\prime} \sigma^{\prime}}\left(1-f_{e k \sigma}\right) g_{N S_{z}^{\prime}}\right],
\end{aligned}
$$

where $f_{l k \sigma}=f_{l}\left(\varepsilon_{l k \sigma}\right)=\left\{1+\exp \left[\left(\varepsilon_{l k \sigma}-\mu_{l}\right) / k_{B} T\right]\right\}^{-1}$ is the Fermi distribution function with the chemical potential $\mu_{l}$, and $g_{N S_{z}}$ is the probability of finding the $N$-electron state with $z$ component of spin $S_{z}$, given by $g_{N S_{z}}=\exp \left[-\left(E_{N S_{z}}\right.\right.$ $\left.-N \mu) / k_{B} T\right] / Z_{d}$ with $Z_{d}$ given by $\Sigma_{S_{z}} g_{N, S_{z}}+\Sigma_{\tilde{S}_{z}} g_{N+1, \tilde{S}_{z}}=1$.

In the absence of spin-orbit interactions, dependences on $\sigma, S_{z}, \widetilde{S}_{z}$ of matrix elements of $H_{\mathrm{t}}$ are expressed using the Clebsch-Gordan coefficient since

$$
\left\langle N, S S_{z}\left|c_{n \sigma}\right| N+1, \widetilde{S} \widetilde{S}_{z}\right\rangle=\left\langle S S_{z} \frac{1}{2} \frac{\sigma}{2} \mid \widetilde{S} \widetilde{S}_{z}\right\rangle h_{n N S \widetilde{S}}
$$

with $h_{n N S \tilde{S}}$ independent of $\sigma, S_{z}, \widetilde{S}_{z}$. We neglect $k$ dependence of $V_{l k n}$ and the energy dependence of the density of states (per spin) $\rho_{l}$ in lead $l$ in its narrow range of interest (of the order of the spin splitting and the thermal energy) and introduce constants describing the level broadening of the many-body states:

$$
\Gamma_{l N S \tilde{S}}=2 \pi \rho_{l}\left|\sum_{n} V_{l n} h_{n N S \tilde{S}}\right|^{2} .
$$

Finally the conductance $G$ for the resonant-tunneling regime $\left(\left|\mu+E_{i}-E_{m}\right|<k_{B} T\right.$ or $\left.\sim k_{B} T\right)$ is expressed as

$$
G=C_{G} F_{0}\left(S, \widetilde{S}, \frac{\mu-\varepsilon_{0}}{k_{B} T}, \frac{g^{*} \mu_{B} B}{k_{B} T}\right)
$$

with a universal function

$$
\begin{aligned}
F_{0}= & \sum_{\sigma S_{z}}\left|\left\langle S S_{z} \frac{1}{2} \frac{\sigma}{2} \mid \tilde{S} S_{z}+\frac{\sigma}{2}\right\rangle\right|^{2} \\
& \times\left(g_{N, S_{z}}+g_{N+1, S_{z}+\sigma / 2}\right)\left(-4 k_{B} T\right) f^{\prime}\left(\varepsilon_{\sigma}\right)
\end{aligned}
$$

and a prefactor

$$
C_{G}=\frac{e^{2}}{\hbar} \frac{1}{4 k_{B} T} \frac{\Gamma_{e N S \tilde{S}} \Gamma_{c N S \tilde{S}}}{\Gamma_{e N S \tilde{S}}+\Gamma_{c N S \tilde{S}}} .
$$

The addition energy $\varepsilon_{\sigma}$ is given by $\varepsilon_{\sigma}=E_{N+1, S_{z}+\sigma / 2}-E_{N, S_{z}}$ $=\varepsilon_{0}+g^{*} \mu_{B} B \sigma / 2$ and $\varepsilon_{0}$ is that in the absence of the magnetic field. The prefactor $C_{G}$ depends on tunneling matrix elements and the many-body ground states with $N$ and $N$ +1 electrons. Because of the rule for the addition of two spins, we have $\widetilde{S}=S \pm 1 / 2$. We only discuss behaviors for $\widetilde{S}$ $=S+1 / 2$ below, since those for $\widetilde{S}=S-1 / 2$ are obtained from $(2 S+1) F_{0}(S, \widetilde{S}, y, b)=(2 \widetilde{S}+1) F_{0}(\widetilde{S}, S,-y,-b) \quad$ with $\quad y$ $=\left(\mu-\varepsilon_{0}\right) / k_{B} T$ and $b=g * \mu_{B} B / k_{B} T$.

Figure 1(a) presents the normalized conductance $F_{0}$ as a function of the chemical potential for several values of the spin splitting for $S=0, \widetilde{S}=1 / 2$. The largest magnetic field in the figure $\left(b=g * \mu_{B} B / k_{B} T=5\right)$ corresponds to $B \sim 1 \mathrm{~T}$ at the temperature of $T=0.1 \mathrm{~K}$ at which the thermallybroadened resonant tunneling is possible to observe. ${ }^{13}$ Increasing the spin splitting, the peak shifts to the lower chemical potential, and its height decreases (the width of the peak is determined by the thermal energy $k_{B} T$ ). The result for $S$ $=0, \widetilde{S}=1 / 2$ is shown to be identical to that of the GlazmanMatveev theory for a single orbit, even for quantum dots with many orbits.

The peak height of $F_{0}$ in the absence of the spin splitting, given by $F_{0}=4 /(1+2 \sqrt{r}+r)$ with $r=(2 S+1) /(2 \widetilde{S}+1)$, represents the current suppression due to the correlation in tunnelings of electrons, since the conductance $F_{0}$ is normalized so that $F_{0}=1$ at the peak in the limit of large spin splitting. In the case of $S=0, \widetilde{S}=1 / 2$ [Fig. 1(a)], the peak height at $B=0$ is $F_{0}=8 /(3+2 \sqrt{2})=1.37$ and is smaller than the value of 2 in the absence of the Coulomb interactions as 
(a) Normalized Conductance $F_{0}$

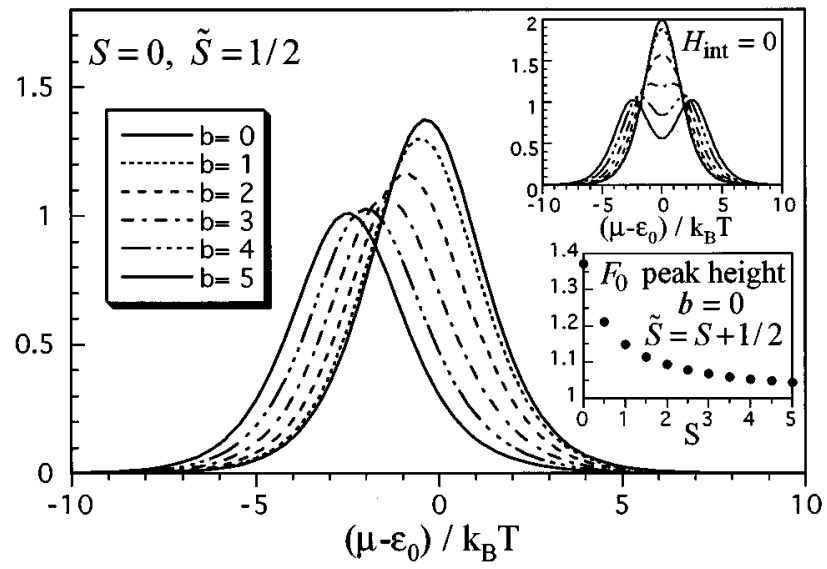

(b) Normalized $\mathrm{AB}$ amplitude $\left|F_{1}-i F_{0}\right|$

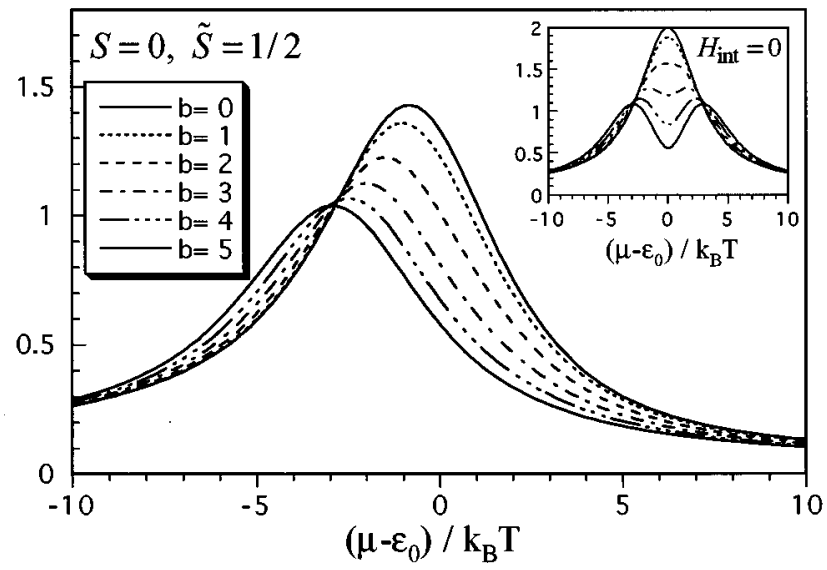

FIG. 1. (a) Normalized conductance $F_{0}$ through a quantum dot as a function of the chemical potential $\mu$ and the spin splitting $b$ $=g^{*} \mu_{B} B / k_{B} T$ for $S=0$ and $\widetilde{S}=1 / 2(S$ and $\widetilde{S}$ are the spins in $N$ and $N+1$-electron ground states, respectively). Upper inset shows the result in the absence of interactions, and the lower inset shows the peak height at $B=0$ as a function of $S(\widetilde{S}=S+1 / 2)$. (b) Normalized amplitude of the $\mathrm{AB}$ oscillations $\left|F_{1}-i F_{0}\right|$.

shown in the upper inset of Fig. 1(a) (the conductance is again normalized by the peak height at $B \rightarrow \infty)$. Interestingly, the peak height at $B=0$ decreases further with $S$ and approaches unity at $S \rightarrow \infty$ as shown in the lower inset of Fig. 1(a). This is due to the larger transition rate for the spin $\sigma$ parallel to the spin of the dot, as expressed by the ClebschGordan coefficient in Eq. (8). Note also that the line shape at $B=0$ is not symmetrical, and the peak position $\mu_{\text {peak }}$ is a little lower than $\varepsilon_{0}:\left(\mu_{\text {peak }}-\varepsilon_{0}\right) / k_{B} T=(1 / 2) \ln r$.

Next, we derive the amplitude of the Aharonov-Bohm (AB) conductance oscillations in the double-slit interferometer. ${ }^{15}$ In this device, a quantum dot is inserted in one of two slits, and the transmission coefficient through the quantum dot is deduced from the $\mathrm{AB}$ oscillations. The coherent component of the conductance through the interferometer is

$$
G_{\mathrm{coh}}=\frac{e^{2}}{h} \sum_{\sigma} \int d \varepsilon\left(-\frac{\partial f}{\partial \varepsilon}\right)\left|t_{0}+t_{\sigma}(\varepsilon) e^{i \varphi}\right|^{2}
$$

Here $t_{\sigma}(\varepsilon)$ is the transmission coefficient through the slit with the dot for an electron with spin $\sigma, t_{0}$ is that through the other slit, and $\varphi=2 \pi \Phi / \Phi_{0}\left(\Phi_{0}=h c / e\right)$ is the phase difference between the two paths due to the magnetic flux $\Phi$ between them. Neglecting the energy dependence of $t_{0}$ within the width of $k_{B} T$ and $g^{*} \mu_{B} B$, the amplitude of the AB oscillations is proportional to $\left|a_{t}\right|$ with

$$
a_{t}=\sum_{\sigma} \int d \varepsilon\left(-\frac{\partial f}{\partial \varepsilon}\right) t_{\sigma}(\varepsilon) .
$$

The transmission coefficient $t_{\sigma}(\varepsilon)$ is given ${ }^{10}$ by

$$
t_{\sigma}(\varepsilon)=2 \pi\left(\rho_{e} \rho_{c}\right)^{1 / 2} \sum_{n n^{\prime}} V_{e n} G_{n n^{\prime} \sigma}(\varepsilon) V_{c n^{\prime}}^{*},
$$

and the retarded Green function $G_{n n^{\prime} \sigma}(\varepsilon)$ is calculated in the noncrossing approximation in the weak-tunneling regime. Then $a_{t}$ is expressed again by universal functions

$$
a_{t}=C_{t}\left(F_{1}-i F_{0}\right),
$$

where one of them is defined by

$$
\begin{aligned}
F_{1}= & \sum_{\sigma S_{z}}\left|\left\langle S S_{z} \frac{1}{2} \frac{\sigma}{2} \mid \tilde{S} S_{z}+\frac{\sigma}{2}\right\rangle\right|^{2} \\
& \times\left(g_{N, S_{z}}+g_{N+1, S_{z}+\sigma / 2}\right) \frac{(-4)}{\pi} g\left(\frac{\mu-\varepsilon_{\sigma}}{k_{B} T}\right)
\end{aligned}
$$

with

$$
g(y)=-\lim _{\delta \rightarrow 0} \int_{-\infty}^{\infty} d x \frac{x}{x^{2}+\delta^{2}} \frac{\exp (x-y)}{[\exp (x-y)+1]^{2}},
$$

and the prefactor is

$$
C_{t}=\frac{\pi^{2} \sqrt{\rho_{e} \rho_{c}}}{2 k_{B} T}\left(\sum_{n} V_{e n} h_{n N S \tilde{S}}\right)\left(\sum_{n} V_{c n} h_{n N S \tilde{S}}\right)^{*} .
$$

Figure 1(b) presents the normalized $\mathrm{AB}$ amplitude $\left|F_{1}-i F_{0}\right|$. The amplitude is again normalized so that the peak height becomes unity in the limit of large spin splitting. The reduction of the peak height at $B=0$ due to the interaction is remarkable. Such reduction of the coherent current is due to the correlation in tunnelings as well as the dephasing by spin flips.

Such an effect of spin flips at $B=0$ is clearly seen in the off-resonance regime $\left(\left|\mu-\varepsilon_{0}\right| \gg k_{B} T\right)$ in which the correlation in tunnelings is not important. The conductance in this regime behaves as $G(\mu)=b_{G}\left(\mu-\varepsilon_{0}\right)^{-2}$ both in $\mu>\varepsilon_{0}$ and in $\mu<\varepsilon_{0}$ with the common prefactor $b_{G}$. On the other hand, the $\mathrm{AB}$ amplitude $\left|a_{t}\right|$, from Eq. (13), behaves as

$$
\begin{aligned}
\left|a_{t}\right| & =b_{t}(2 \widetilde{S}+1)^{-1}\left|\mu-\varepsilon_{0}\right|^{-1}, & \mu>\varepsilon_{0} \\
& =b_{t}(2 S+1)^{-1}\left|\mu-\varepsilon_{0}\right|^{-1}, \quad & \mu<\varepsilon_{0} .
\end{aligned}
$$

The reduction of $\left|a_{t}\right|$ by the spin degeneracy is due to spin flips at the dot. Such dephasing is most prominent at $S=0$ and $\widetilde{S}=1 / 2$ where $\left|a_{t}\right|$ in $\mu>\varepsilon_{0}$ is reduced by $1 / 2$ compared with that in $\mu<\varepsilon_{0}$, which is evident in Fig. 1(b). At large spin splittings, the spin flip is absent and both the conduc- 
tance and the $\mathrm{AB}$ amplitude are symmetrical with respect to the peak position $\mu=\varepsilon_{\sigma}$ with $\sigma / 2=\widetilde{S}-S$.

The generalization to materials with the valley degree of freedom $v$ is straightforward: change as $k \rightarrow v k$ (let $n$ label all single-particle states in the dot). The above formulas for the conductance and the $\mathrm{AB}$ amplitude are still applicable if we redefine the constants as

$$
\Gamma_{l N S \tilde{S}}=\sum_{v} 2 \pi \rho_{l v}\left|\sum_{n} V_{l v n} h_{n N S \tilde{S}}\right|^{2},
$$

and

$$
C_{t}=\sum_{v} \frac{\pi^{2} \sqrt{\rho_{e v} \rho_{c v}}}{2 k_{B} T}\left(\sum_{n} V_{e v n} h_{n N S \tilde{S}}\right)\left(\sum_{n} V_{c v n} h_{n N S \tilde{S}}\right)^{*} .
$$

In conclusion, we have derived universal dependences on the spin splitting of the conductance and the transmission coefficient through a quantum dot with many levels. The universality is due to the symmetry in the spin space in the absence of spin-orbit interactions. Fitting experimental data with the universal function will give the magnitude of the spin in the initial state and the intermediate state and, more importantly, a clear evidence of the correlation in tunnelings and the dephasing due to spin flips. The in-plane magnetic field, together with the widely-used temperature scan, will be a useful tool in studying many-body effects, which appear in the height and the line shape of resonant-tunneling peaks. The spin blockade studied theoretically by several authors $^{22,16,23-25}$ will be another testing ground for the usefulness of the in-plane magnetic field.

The author would like to thank T. Ando, Y. Iye, S. Katsumoto, N. Tokuda, and Y. Asano for valuable discussions. This work was supported in part by a Grant-in-Aid for Scientific Research on priority area "Single Electron Devices and Their High Density Integration', from the Ministry of Education, Science, Sports and Culture, Japan.
${ }^{1}$ M. A. Kastner, Phys. Today 46 (1), 24 (1993); Rev. Mod. Phys. 64, 849 (1992).

${ }^{2}$ S. Tarucha, D. G. Austing, T. Honda, R. J. van der Hage, and L. P. Kouwenhoven, Phys. Rev. Lett. 77, 3613 (1996).

${ }^{3}$ L. I. Glazman and K. A. Matveev, Pis'ma Zh. Éksp. Teor. Fiz. 48, 403 (1988) [JETP Lett. 48, 445 (1988)].

${ }^{4}$ D. Ephron, Y. Xu, and M. R. Beasley, Phys. Rev. Lett. 69, 3112 (1992).

${ }^{5}$ H. Akera, Phys. Rev. B 47, 6835 (1993); Jpn. J. Appl. Phys., Part 1 36, 3974 (1997); Solid-State Electron. 42, 1379 (1998); Jpn. J. Appl. Phys., Part 1 38, 384 (1999).

${ }^{6}$ A. Levy Yeyati and M. Büttiker, Phys. Rev. B 52, R14 360 (1995).

${ }^{7}$ G. Hackenbroich and H. A. Weidenmüller, Phys. Rev. Lett. 76, 110 (1996).

${ }^{8}$ C. Bruder, R. Fazio, and H. Schoeller, Phys. Rev. Lett. 76, 114 (1996).

${ }^{9}$ P. Singha Deo and A. M. Jayannavar, Mod. Phys. Lett. B 10, 787 (1996).

${ }^{10}$ Y. Oreg and Y. Gefen, Phys. Rev. B 55, 13726 (1997).

${ }^{11}$ W. Izumida, O. Sakai, and Y. Shimizu, J. Phys. Soc. Jpn. 66, 717 (1997).
${ }^{12}$ M. A. Davidovich, E. V. Anda, J. R. Iglesias, and G. Chiappe, Phys. Rev. B 55, R7335 (1997).

${ }^{13}$ A. Yacoby, M. Heiblum, D. Mahalu, and Hadas Shtrikman, Phys. Rev. Lett. 74, 4047 (1995).

${ }^{14}$ S. Katsumoto and A. Endo, J. Phys. Soc. Jpn. 65, 4086 (1996); Jpn. J. Appl. Phys., Part 1 36, 3978 (1997).

${ }^{15}$ R. Schuster, E. Buks, M. Heiblum, D. Mahalu, V. Umansky, and H. Shtrikman, Nature (London) 385, 417 (1997).

${ }^{16}$ Y. Tanaka and H. Akera, Phys. Rev. B 53, 3901 (1996).

${ }^{17}$ Y. Meir and N. Wingreen, Phys. Rev. Lett. 68, 2512 (1992).

${ }^{18}$ C. W. J. Beenakker, Phys. Rev. B 44, 1646 (1991).

${ }^{19}$ D. V. Averin, A. N. Korotkov, and K. K. Likharev, Phys. Rev. B 44, 6199 (1991).

${ }^{20}$ Y. Kuramoto, Z. Phys. B 53, 37 (1983).

${ }^{21}$ N. E. Bickers, Rev. Mod. Phys. 59, 845 (1987).

${ }^{22}$ D. Weinmann, W. Häusler, and B. Kramer, Phys. Rev. Lett. 74, 984 (1995)

${ }^{23}$ M. Eto, J. Phys. Soc. Jpn. 66, 2244 (1997).

${ }^{24}$ H. Imamura, H. Aoki, and P. A. Maksym, Phys. Rev. B 57, R4257 (1998).

${ }^{25}$ Y. Asano, Phys. Rev. B 58, 1414 (1998); J. Phys. Soc. Jpn. 67, 4014 (1998). 